\title{
Editorial
}

\section{"RELEASE FROM CONTROL" IN LEPROSY}

Up till 10 years ago the "criteria for discharge" in leprosy were regarded as a logical, necessary and relatively clear-cut aspect of patient care. The menacing psychological atmosphere which surrounded leprosy, both for patient and doctor alike, gave enormous importance to the concept of an end-point, when after prescribed courses of chemotherapy, and the attainment of a condition of "inactivity", a further period of observation and treatment could lead to the day when the disease would be regarded as arrested, and, hopefully, overcome. At that point chemotherapy could be suspended, a medical certificate given, and with the safeguard of regular but occasional follow-up, the patient could be restored to normal life, This was a day of great emotional content for patients.

During the past decade several aspects of this approach to leprosy have been challenged by events and by better understanding of the bacteriology and immunology of Mycobacterium leprae. The idea that a line can be drawn between infection and no infection has been rudely shattered. The very natural bacillus-orientated approach made it easy to pontificate and even legislate on behalf of patients in the belief that they would meekly follow our advice. We have had to learn that the final arbiter on how much chemotherapy is taken and when treatment is stopped is not the doctor, but the patient himself. All our well laid plans and carefully considered judgments fall to the ground if the patient on whose behalf they are made simply fails to co-operate. He, as well as the germ, must be at the centre of our concern, and our approach be such that our well meant programmes are not frustrated.

The judgments of the WHO Expert Committee on Leprosy on this subject are highly relevant. The Report of the 4th Expert Committee (1970) included the following:

"A leprosy patient without any sign of clinical activity, and with negative bacteriological findings should be considered as an 'inactive case'.

Once inactivity is achieved, regular treatment should be continued for varying periods of time before a patient is 'released from control' ( $\mathrm{rfc}$ ). These periods should be $1 \frac{1}{2}$ years for tuberculoid, 3 years for indeterminate, and at least 10 years for lepromatous and borderline cases. Since data on relapse after rfc are scarce, it is advisable and important to continue the follow-up of lepromatous cases but without treatment. Some leprologists consider that this should be done for life." 
This statement was endorsed by the 5th Expert Committee (1976) with an interesting proviso. "The Committee strongly recommended that inactive tuberculoid and indeterminate cases be promptly released when they meet the criteria. This action is not only in the interest of the patient but has an important bearing on operational efficiency and would release resources for other activities of the programme."

Clearly in 1976 wider issues came within the purview of the Committee than the earlier approach, but the endorsement of the 1970 statement means that the views then expressed are carried forward as the recommended norm for today. Some aspects of this invite discussion.

It must first be said that for patients with types of leprosy towards the tuberuloid end of the spectrum the traditional approach remains valid. With such patients the question of ostracism and isolation should not have arisen, and following chemotherapy, inactivity and continuing therapy for the times suggested by the Committee, an end-point can be envisaged and a discharge certificate be given with minimal prospect of relapse. The key here is surely the existence of cell-mediated immunity as the limiting factor in the infection, holding the promise of continuing ability to deal with the bacillus, and monitored by a positive reaction to lepromin, whether this was there from the start or develops in association with reversal reactions.

\section{Continuing Chemotherapy and Surveillance}

When we turn to patients with bacilliferous leprosy the position is rendered much more difficult by the discovery that even in spite of long periods of chemotherapy viable $M$. leprae may persist, notably in the skin, bone marrow, lymph glands and nerves. Clearly it is very important that the types of patient in whom such persister bacilli are found should be identified as accurately as possible. At present they must be assumed to include all patients with established lepromatous leprosy. How far this phenomenon extends into the borderline part of the spectrum remains to be determined. The crucial question is the extent to which such persister bacilli are responsible for relapse. A lot more data are needed on the whole subject, but there seems to be no logical reason why persister bacilli, granted favourable conditions, should not recommence reproductive life, the only possible exception being the small minority of such patients who ultimately attain lepromin positivity.

It is not difficult to envisage such favourable conditions. We already encounter them in relation to downgrading reactions. Clearly the discovery of persister bacilli has an important bearing on chemotherapy. With such patients there can surely be no magical moment when after 10 years of inactivity we can say, "Now it is all over, you can stop taking dapsone". At present the only defence we have against relapse is continuous chemotherapy. As long as we are dependent on dapsone for maintenance therapy it would seem to be obvious advice to the patient with LL or BL leprosy that he should continue to take dapsone at therapeutic dosage indefinitely, even though he appears to be clinically inactive. This means that in present circumstances there should be no discharge from treatment for such patients, and the "criteria for discharge" have become irrelevant. 
By the same token there can be no discharge from regular surveillance. The whole purpose of surveillance is to monitor the possibility of relapse, whether this is caused by dapsone-sensitive or dapsone-resistant bacilli. As long as we accept the reasonable possibility of relapse, then surveillance must continue indefinitely, and is indeed very important.

It would appear therefore that in LL and BL leprosy, with present chemotherapy and with present day knowledge, discharge from treatment or from surveillance should not be advised, both in the best interests of the patient and of the community.

\section{The Personal Factor}

Acceptance of this principle immediately poses a difficult question: How can we expect the co-operation of patients in such procedures? Careful studies in recent years have indeed exposed the truth that frequently only a minority of patients continue to take dapsone as prescribed [e.g. Malawi: Ellard, Gammon and Harris (1974); Ethiopia: Low and Pearson (1974); Bombay: Naik (1977)]. The same applies to attendances at treatment clinics. Many patients after longer or shorter periods of attendance simply discharge themselves and make nonsense of the medical criteria for discharge. It so happens that patients with $\mathrm{LL}$ and BL leprosy are often the most faithful in their attendance, but there is a problem here which must be resolved.

Now it is a fact of medical experience that people in general like to take medicines, whether they are Africans, Asians or Europeans, and will continue to do this as long as they feel that prescribed medicines are doing them good, the medicines are easy to obtain, and there are no economic or social problems involved. The taking of tablets indefinitely holds no intrinsic problem. Millions of people do it, e.g. for hypertension, rheumatic conditions, or diabetes. Exactly the same is true of periodic medical examinations, provided the doctor-patient relationship is what it ought to be. Why then do we expect something different where leprosy is concerned? The reason cannot lie simply in the need for protracted treatment. It lies in the way the patient regards his illness and in the way he feels that other people, including the doctor, regard him, the sufferer from leprosy. All too of ten in the minds of patient, community and doctor alike, leprosy continues to hold a special anxietycreating position, the natural reaction to which is to turn away from it, fail to face it, or forget it as quickly as possible.

The WHO Committee recommends 10 years of chemotherapy after inactivity in bacilliferous leprosy. If a patient has persisted for 10 years, he must so have got into the habit that there should not be the slightest difficulty in his taking it for 11,12 years or indeed indefinitely. This is not the problem. The real problem is the desire to escape from the association with leprosy. This is something that deserves much more careful consideration than is usually given to it.

The best likelihood of maintaining continuity of treatment and surveillance, of encouraging the patient to persevere, will occur if three things are safeguarded. 
(1) The patient must be helped to understand the nature of his illness.

(2) The doctor-patient relationship must be good. So much depends on this. It is the experience of the writer that where patients believe they are going to be welcomed and treated with understanding and consideration they are prepared to face the facts of their disease and respond with continuous co-operation.

(3) Dapsone must be available as simply and unobtrusively as possible, not in the context of time consuming frequent visits to special clinics labelled in everyone's minds as reserved for people with active leprosy.

These principles apply during the first 10 years as well as subsequently. If they can be achieved there seems to be no logical reason why a patient should not of his own choice, continue to remain under chemotherapy and surveillance indefinitely.

It appears to the writer that the sustained co-operation of patients with LL and BL leprosy is made unnecessarily difficult by the perpetuation of old ideas and emphases.

The WHO definition of inactivity includes negative bacteriological findings, a feature which condemns many patients at the lepromatous end of the spectrum to long years of carrying the anxiety, and usually the stigma, of active leprosy. The traditional attitude would maintain that as long as acid-fast material, regardless of its morphology, is found in routine skin smears, the patient is still suffering from active disease, and by inference other people are at risk.

Ever since the work of Rees and Valentine (1962), the judgment has continuously built up at centres of the highest excellence in leprosy research, that viability in $M$. leprae is associated with the intact rod-shaped, uniformly staining organism. Various attempts have been made to cultivate $M$. leprae from fragmented bacillary material. None has been authenticated.

If we are ready to accept that it is the morphologically intact normal staining form of the bacillus which is responsible for the disease leprosy and for transmitting that disease, then any basic anxiety we have concerning leprosy as a transmissible disease should centre around that form of the bacillus and not around dead fragmented forms of no significance in the transmission of the disease. The role of dead bacilli in relation to ENL and neurological aspects of leprosy is an important but quite different question. From the angle of the patient's capacity to transmit the disease, can it not be generally accepted that if the morphological index is zero, i.e. no bacilli of intact shape and staining can be found on careful bacteriological examination, then the numbers of such bacilli are so slight, that as with tuberculosis the patient may be regarded as non-infective to others. Persister bacilli in deep organs are not capable of leaving the body and have no relevance to this matter. The important point is that if the patient is in practice regarded as not infective to others, then we should say so. This would give an enormous boost to the morale of patients with these types of leprosy. So often the consciousness of being infective to others creates deep anxiety in the minds of patients where their children are concerned, and is a potent factor in the 
depression that so easily leads to despair and non-co-operation. Furthermore, the logic of the situation should be followed through. The patient should surely be regarded as in no way different from any other sick person. He should be able to attend general out-patient's departments, occupy any appropriate hospital bed, be employed in any suitable capacity and have no social restrictions placed on him whatever. The idea that these normal prerogatives of people in community are to be denied until the traditional routine smears are totally negative for any acid fast-material is unscientific and indeed may even be considered uncharitable. This suggestion is not inherent in the WHO statement, but it is very much the common interpretation of what "release from control" really means, an echo of earlier rigid attitudes to this disease.

\section{A Liberal Outlook Must be Backed by Sound Technology}

If we are looking diligently for viable bacilli, our technology must be reliable. This means first, that the nose must be included in our attention. This is still frequently neglected, or if included, nasal examination often takes the form of old-fashioned septal smears, taken less than $2 \mathrm{~cm}$ within the anterior nares and calculated to yield nothing important. The inferior turbinate must engage our attention, and if as frequently happens it does not seem at first glance to be there, we need to realise that its anterior end has already been eroded by serious lepromatous disease. A bacteriological examination of the nasal discharge, choosing saneo-purulent areas is a sine qua non.

Secondly, relapse commonly first manifests itself by the appearance at maybe a single site in the skin of large numbers of normal-staining bacilli in a patient elsewhere and previously exhibiting only fragmented bacilli. Three months later normal viable bacilli are likely to be widespread, but by then it is certain that intranasal inf ection will have been re-activated, maybe worse than before initial treatment, and the patient be already discharging large numbers of viable bacilli from the nose. Early discovery of relapse is thus extremely important. There is no guarantee that common methods of skin smears, selecting fixed sites in an inflexible routine, will identify relapse in its earliest stages, though careful clinical examination might well have aroused suspicion. This is particularly important if the relapse as is very common, takes the form of histoid lesions. An important feature of histoid leprosy is its capacity to appear in areas of the body not usually selected for routine bacteriological examination, e.g. the buttocks, lower abdomen, upper thighs and genitalia. Histoid lesions readily ulcerate and discharge enormous numbers of viable bacilli. To the uninitiated a well defined histoid lesion might even be mistaken for a reversal reaction or unusual form of ENL. Clinical acumen and skill in taking and reading smears are essential if relapse is to be detected early. Failure on either side brings surveillance into disrepute and leads to the pessimistic dictum that inactivity in lepromatous leprosy cannot be accurately assessed by routine bacteriological methods. There is comfort in the fact that relapse is far more common among patients who are not taking regular chemotherapy than among those who are, and this particularly applies to relapse with histoid leprosy. 


\section{Wider Issues}

In all our judgments affecting the life and well being of patients, the preservation and protection of the patient's place in community life is a longterm priority and there is no escape from our responsibility in this direction. Some separation during the stage of the disease when the patient is discharging viable bacilli must be acceptable, but as the morphological index is usually zero within 6 months or thereabouts of starting treatment, this is the sort of limit that needs generally to be visualized. There is, of course, no harm in some sort of "disease arrested" certificate following the criteria enunciated by the WHO Committee, but far more important in practice for the patient with LL or BL leprosy would be some form of medical certification given on request when the stage of negative MI had been attained, and indicating that the patient could be regarded as non-infective to others. Such a certificate would indeed release the patient from his primary anxieties and restrictions, encourage his continuing co-operation and promote a much more open and healthy attitude to leprosy generally.

It must be admitted that to secure such conditions considerable reorientation and re-education of both patients and the community are needed, regarding the nature of leprosy, the delivery of chemotherapy, and the meaning of surveillance. It can however be done, as Antia (1977) has shown.

The fear that leprosy may be transmitted by forms of the bacillus other than the intact rod-shaped bacillus may be hypothetical, but it dies hard, especially in relation to leprosy that appears to have been traced to patients considered bacteriologically negative. Davey and Rees (1974) showed that M. leprae could remain viable in air for up to 7 days. Recent work by Desikan (1977) extends this period to 11 days. These findings pinpoint possible unsuspected sources of such infections. The basic plea of this paper is that our sense of responsibility for patients with $L L$ and Bl leprosy should help us to accept with courage the facts regarding the viability of $M$. leprae which have been authenticated, and apply them resolutely.

There is one final point. Is "Release from Control" a phrase worth retaining in its present context? In common English usage "release" suggests a previous state of bondage or imprisonment. "Control" suggests a restriction on movement. Both words, used in relation to patients who have been under chemotherapy for years and exhibited no sign of viable bacilli for a long time are not only irrelevant but psychologically harmful to patients, workers, and community alike. Are they really necessary?

\section{T. F. DAVEY}

\section{References}

Antia, W. H. (1977). Lepr. Rev. 48, 155.

Desikan, K. V. (1977). Lepr. Rev. 48, 231.

Davey, T. F. and Rees, R. J. W. (1974). Lepr. Rev. 45, 121.

Ellard, G. A., Gammon, P. T. and Harris, J. M. (1974). Lepr. Rev. 45, 224.

Low, J. S. H. and Pearson, J. M. H. (1974). Lepr. Rev. 45, 218.

Naik, S. S. (1977). Lepr. Rev. 48, 135.

Rees, R. J. W. and Valentine, R. C. (1962). Int. J. Lepr. 30, 1.

World Health Organization Technical Report Series, No. 459 (1970).

World Health Organization Technical Report Series, No. 607 (1976). 